\title{
Ruin Probabilities in Risk Based on a Generalized FGM Dependence Structure
}

\section{Liyi Wen}

School of Mathematics and Statistics, Chongqing University of Technology, Chongqing, China Email: wenkaihong@hotmail.com

Received 5 May 2016; accepted 19 May 2016; published 24 May 2016

Copyright (C) 2016 by author and OALib.

This work is licensed under the Creative Commons Attribution International License (CC BY).

http: //creativecommons.org/licenses/by/4.0/

(c) $\underset{\mathrm{EY}}{0}$ Open Access

\section{Abstract}

In this paper, we consider a discrete time insurance risk model, in which insurance and financial risks jointly follow a bivariate generalized FGM distribution. Assuming that every convex combination of the marginal distributions of insurance and financial risks belongs to strongly regular variation class, we derive some asymptotic equivalence formulas for these probabilities with both finite and infinite time horizons, all in the form of linear combinations of the tail probabilities of the insurance and financial risks.

\section{Keywords}

Insurance and Financial Risks, Ruin Probabilities, Generalized FGM Distribution, Strongly Regular Variation Class

Subject Areas: Financial Mathematics, Mathematical Economics, Probability Theory

\section{1. 引言}

众所周知, 保险公司的资产在随机经济环境中将会导致两种风险, 通常称其为保险风险和金融风险 [1]。其中，保险风险是因传统保险理赔业务引起的责任险，而金融风险是因保险公司用其持有的部分资 产在金融市场上作风险投资引起的投资风险。在随机经济环境的前提下，根据保险实务和金融市场规律， 需要作如下假设 [1] [2]: 保险公司在周期 $i$ 的保费收入为 $C_{i}>0$ 支出为 $A_{i}>0$ 且 $C_{i}, A_{i}$ 为随机变量; 时间 $i-1$ 到 $i$ 的随机通胀因子为 $B_{i}$; 保险公司的初始资本为 $x(x>0)$, 综上, 记保险公司在有限时间 $n$ 的资本累 积为 $U_{n}$, 则我们可得:

$$
U_{0}=x, \quad U_{n}=x \prod_{i=1}^{n} B_{i}+\sum_{i=1}^{n}\left(C_{i}-A_{i}\right) \prod_{j=1+i}^{n} B_{j} .
$$


为了处理方便, 记 $\prod_{j=1+i}^{i} B_{j}=1$ 。现在引入 $X_{i}=A_{i}-C_{i}$, 则 $X_{i}$ 为保险公司的净损失, 即人们称的保险风 险; 设时间 $i$ 到 $i-1$ 的随机贴现因子为 $Y_{i}$, 则 $Y_{i}=1 / B_{i}$, 也就是人们称的金融风险, 现用 $\hat{U}_{n}$ 表示有限时 间 $n$ 的资本累积 $U_{n}$ 的贴现值, 则

$$
\widehat{U}_{n}=U_{n} \prod_{j=1}^{n} Y_{j}=\left(x \prod_{i=1}^{n} B_{i}-\sum_{i=1}^{n} X_{i} \prod_{j=1+i}^{n} B_{j}\right) \prod_{j=1}^{n} Y_{j}=x-\sum_{i=1}^{n} X_{i} \prod_{j=1}^{i} Y_{j} .
$$

现在假设保险公司的风险投资比例系数为 $p, p \in[0,1], W_{i}$ 表示投资组合的价值, 于是 $W_{i}=p W_{i-1}\left(1+r_{i}\right)+(1-p) W_{i-1}(1+r)$, 则随机累积因子 $B_{i}=W_{i} / W_{i-1}=(1-p)(1+r)+P\left(1+r_{i}\right)$, 从而对应的贴 现因子 (金融风险) $Y_{i}=1 /\left[(1-p)(1+r)+p\left(1+r_{i}\right)\right]$, 这里 $r$ 为利率, $r_{i}$ 为投资回报率 $[2]$ 。

在上式中若 $x<\sum_{i=1}^{n} X_{i} \prod_{j=1}^{i} Y_{j}$, 则称保险公司破产了, 这里的破产只是针对数学范畴而言的。在保险 实务中, 即使保险公司资产为负, 也有机会恢复正常运营。本文感兴趣的是在数学范畴内用概率的方法 来研究有限时间 $n<\infty$ 和无限时间 $n \rightarrow \infty$ 时保险公司发生破产情况, 令 $S_{n}=\sum_{i=1}^{n} X_{i} \prod_{j=1}^{i} Y_{j}$, 那么有限时间 $n$ 的破产概率表示为 $[1]$ :

$$
\psi(x, n)=\operatorname{Pr}\left(x-S_{n}<0\right)=\operatorname{Pr}\left(S_{n}>x\right)
$$

记 $M_{n}=\max _{1 \leq m \leq n} S_{m}>x$, 则有限时间 $n$ 的破产概率表示为:

$$
\psi(x, n)=\operatorname{Pr}\left(\inf _{1 \leq m \leq n}\left(x-S_{m}\right)<0\right)=\operatorname{Pr}\left(\max _{1 \leq m \leq n} S_{m}>x\right)=\operatorname{Pr}\left(M_{n}>x\right) .
$$

同时, 我们可得无限时间 $n \rightarrow \infty$ 的破产概率表示为:

$$
\psi(x)=\lim _{n \rightarrow \infty} \psi(x, n)=\lim _{n \rightarrow \infty} \operatorname{Pr}\left(x-S_{n}<0\right)=\operatorname{Pr}\left(S_{\infty}>x\right)
$$

和

$$
\psi(x)=\lim _{n \rightarrow \infty} \psi(x, n)=\lim _{n \rightarrow \infty} \operatorname{Pr}\left(\max _{1 \leq m \leq n} S_{m}>x\right)=\operatorname{Pr}\left(M_{\infty}>x\right) .
$$

显然, 在 $(1.3)$ 式中 $0 \leq M_{n} \leq \sum_{i=1}^{n}\left(X_{i} \vee 0\right) \prod_{j=1}^{i} Y_{j}$, 如果 $\left\{\left(X_{1}, Y_{1}\right),\left(X_{2}, Y_{2}\right), \cdots\right\}$ 构成的独立同分布的随机对序列 满足 $-\infty \leq E \ln Y_{1}<0$ 和 $E \ln \left(X_{1} \vee 1\right)<\infty$, 则相对应的 $S_{n}, M_{n}$ 是收玫的[3]。

在保险风险的研究中, 对其理赔额的历史数据分析中, 我们发现 “占理赔总次数 $20 \%$ 的那些理赔的 数额之和往往达到历次理赔总额的 $80 \%$, 甚至更多! ” (著名的二八定律)这些以很小概率发生的理赔竟 然对保险公司的业务产生如此巨大的影响, 不能不引起大家的关注。如何用概率方法来刻画和解释这类 现象? 研究应用概率的学者们发现重尾分布族可以很好的解释其理赔事件和理赔额的关系, 重尾分布族 更贴近实际, 因此, 重尾分布族成了保险风险研究的重点、热点之一, 本文所研究的破产概率渐近等价 式也是在重尾分布族这个条件下进行的。事实上，有关重尾分布族的破产概率研究已经取得一些不错的 成果, 如文献[1] [2] [4]-[12], 不过这些学者们大都集中在研究不同重尾分布族时的保险风险与金融风险 独立的情形, 如文献[2] [4]-[6]。这类文章具有典型代表是 Tang \& Tsitsiashvili [1]总结归纳了一些学者的 成果, 在这个基础上研究保险风险和金融风险在 $\mathcal{L} \cap \mathcal{D}$ 族的破产概率问题, 得到了有限时间内的保险公 司破产概率的渐近等价式, 接着其他学者在 Tang \& Tsitsiashvili [1]的基础上对其进行了广泛的研究, 如 文献[4]-[12]等。其中, Zhang [6]设 $\Theta=\prod_{j=1}^{i} Y_{j}$, 于是上述问题就可以简化为研究独立随机变量的随机加权 
和问题, 并且这里 $\Theta$ 可为任意相依结构, 推导出保险风险在 $\mathcal{C}$ 族分布下的有限时间的破产概率渐近等价 式。然而, 上述研究的成果都不能很好的反映保险公司的实际运作情况, 比如在汽车保险中大额的车损 理赔往往带有人员的医疗理赔, 又如金融危机造成的利率、金融市场不稳定等对保险业的影响，也就是 说保险实务中各种风险之间是存在一定关系的, 学者们考虑独立情形是为了数学上处理的方便。因此, 我们需要研究相依风险模型, 目前刻画保险风险相依关系最有效的方法和手段之一是用 Copula 函数 (Ali-Mikhail-Haq, FGM，广义 FGM 等), Copula 函数在刻画相依关系具有以下几个方面的优势[14]: 描 述随机变量相关性只需要从联合分布函数构建; 可以描述随机变量的协同性; 构造多维随机变量分布函 数等, 比如下文中介绍的文献[7]-[12]等都采用了 Copula 函数方法。其中, Chen [7]研究了保险风险与金 融风险在 $\mathcal{S}, \mathcal{R}_{-\alpha}$ 族分布下的破产概率，她假设保险风险与金融风险之间构成独立同分布的随机对序列且 满足 FGM 分布(系数 $\theta$ 取值范围为 $(-1,1])$, 她得到了有限时间下的破产概率一般渐近等价式并给出了具 体的、严谨的证明。Chen [8]在她 Chen [7]的基础上对其做了进一步完善, 考虑 FGM 相依分布中系数 $\theta=-1$ 的特殊情形, 获得了与她 Chen [7]相类似的结论。事实上, 国内学者 Jiang \& Tang [10]发表的论文中早已 经研究了基于广义 FGM 分布的保险风险与金融风险相依问题, 并获得了类似于 Chen (2011) 的结果, 只 是由于某些原因而没有获得广泛关注。最近，Li \& Tang [11]考虑保险风险与金融风险构成的凸组合是强 正则变化族 $\left(\mathcal{R}_{-\alpha}^{*}\right)$ 下, 研究了独立和 FGM 相依情形的破产概率, 得到了在一个假设条件下(下文中假设 a)的有限和无限时间的破产概率的渐近等价式。

本文在 $\mathrm{Li} \& \mathrm{Tang}[11]$ 等基础上, 考虑由保险风险 $X$ 与金融风险 $Y$ 构成的凸组合是强正则变化族 $\left(\mathcal{R}_{-\alpha}^{*}\right)$, 在广义 FGM 分布的条件下, 推导出有限时间和无限时间的破产概率渐近等价式, 推广了 Li \& Tang [11]的研究成果。接下来的文章的结构安排如下, 第二部分介绍一些准备工作和引理, 第三部分是主要结 论以及一些证明过程。

\section{2.准备工作和引理}

本文在没有特殊说明的情况下, 以下关系式都表示 $x \rightarrow \infty$ 时成立。设两个正函数 $f(x), g(x)$ : 如果 $\lim _{x \rightarrow \infty} \sup f(x) / g(x) \leq 1$ 成立, 记: $f(x) \lesssim g(x)$ 或者 $g(x) \gtrsim f(x)$; 如果 $f(x) \lesssim g(x)$ 且 $f(x) \gtrsim g(x)$ 成 立, 记: $f(x) \sim g(x)$; 如果 $f(x)=O(g(x))$ 且 $g(x)=O(f(x))$, 记: $f(x) \asymp g(x)$; 除此之外, 对实数 $x$, 记 $x_{+}=x \vee 0$ 和 $x_{-}=-(x \wedge 0)$ 。

\section{1. 重尾分布族}

定义 2.1: (重尾分布 [12]) 在 $\mathbb{R}$ 上的分布 $F$, 如果对任意的 $\alpha>0$ 有 $\int_{-\infty}^{\infty} \mathrm{e}^{\alpha x} F(\mathrm{~d} x)=\infty$, 则称分布 $F$ 是重 尾的, 记作 $F \in \mathcal{K}$ 。

定义 2.2: $\left(\mathcal{S}(\alpha)\right.$ 族[13])在 $[0, \infty)$ 上的分布 $F$, 如果对 $\alpha>0$ 有 $\lim _{x \rightarrow \infty} \frac{\overline{F^{2^{*}}}(x)}{\bar{F}(x)}=2 c<\infty, c=E \mathrm{e}^{\alpha X}$ 且对所 有的 $t \in \mathbb{R}$, 有 $\lim _{x \rightarrow \infty} \frac{\overline{F(x-t)}}{\bar{F}(x)}=\mathrm{e}^{\alpha t}$, 则称分布 $F$ 是卷积等价的(Convolution-equivalent), 记作 $\mathcal{F} \in \mathcal{S}(\alpha)$ 。

定义 2.3: ( $\mathcal{R}_{-\alpha}$ 族 $\left.[13]\right)$ 在 $\mathbb{R}$ 上的分布 $F$, 如果对任意的 $y>0$ 有 $\lim _{x \rightarrow \infty} \frac{\bar{F}(x y)}{\bar{F}(x)}=y^{-\alpha}, \alpha \geq 0$, 则称分布 $F$ 是正则变化尾的(Regularly varying tailed), 记作 $\mathcal{F} \in \mathcal{R}_{-\alpha}$ 。

定义 2.4: ( $\mathcal{R}_{-\alpha}^{*}$ 族 [11]) 在 $\mathbb{R}$ 上的分布 $F$, 如果对任意的 $x \in \mathbb{R}$ 有 $V(x)=1-\frac{\bar{F}\left(e^{x}\right)}{\bar{F}(0)}$ 属于 $\mathcal{S}(\alpha)(\alpha \geq 0)$, 
则称分布 $F$ 是强正则变化尾的(Strongly regular variation tailed), 记作 $\mathcal{F} \in \mathcal{R}_{-\alpha}^{*}$ 。

根据定义 2.2-2.4，有 $\mathcal{R}_{-\alpha}^{*} \subset \mathcal{R}_{-\alpha} \subset \mathcal{S}(\alpha)$ 成立 $[11]$, 更多关于重尾分布族的定义性质可阅读文献资料 [13]等。

\section{2.广义 FGM 分布}

设随机向量 $(X, Y)$, 具有如下形式的联合分布函数:

$$
\Pi(x, y)=F(x) G(y)[1+\theta A(F(x)) B(G(y))]
$$

其中, $\theta \in[-1,1], F, G$ 是随机变量 $X, Y$ 的边缘分布函数, 这里 “核” 函数 $A(x), B(y)$ 满足 $\lim _{x \rightarrow 1} A(x)=0$, $\lim _{y \rightarrow 1} B(y)=0$ 和一些正则性条件, 使得 $\Pi(x, y)$ 为适当的二维分布。我们称(2.1)式表示的分布为广义 Farlie-Gumbel-Morgenstern 分布, 简称为广义 FGM 分布[10]。根据 Nelsen [14]的研究, 记:

$$
C(u, v)=u v[1+\theta A(u) B(v)]
$$

称(2.2)式为广义 FGM Copula 函数。若 $A(u)=1-u, B(v)=1-v$, 则(2.2)式就是我们熟知的标准的 FGM Copula 函数, 根据 2.1 式知边缘分布的相依程度取决于参数 $\theta$ 和函数 $A(\cdot), B(\cdot)$ 。

\section{3. 若干引理}

接下来我们需要介绍几个引理, 这几个引理在下文定理的证明中起着至关重要的作用, 有关这些引 理的详细证明可以阅读文献[11]。

引理 2.1: 设在 $\mathbb{R}$ 上的分布 $F_{1}, \cdots, F_{n}$, 如果对每一个 $q \in \Delta, \Delta=\left\{\left(q_{1}, \cdots, q_{n}\right) \in(0,1)^{n}: \sum_{i=1}^{n} q_{i}=1\right\}$, 有 $F_{q}=\sum_{i=1}^{n} q_{i} F_{i} \in R_{-\alpha}^{*}, \alpha \geq 0$, 则 $F_{1} * \cdots * F_{n} \in \mathcal{S}(\alpha)$ 且

$$
\overline{F_{1} * \cdots * F_{n}} \in \mathcal{S}(\alpha) \sim \sum_{i=1}^{n}\left(\prod_{j=1, j \neq i}^{n} \hat{F}_{j}(\alpha)\right) \bar{F}_{i}(x) .
$$

引理 2.2: 设 $X_{1}, \cdots, X_{n}(n \geq 2)$ 是独立非负的随机变量, $X_{1}, \cdots, X_{n}$ 对应的分布分别为 $F_{1}, \cdots, F_{n}$ 。如果 对每个 $q \in \Delta$ 有 $F_{q}=\sum_{i=1}^{n} q_{i} F_{i} \in R_{-\alpha}^{*}, \alpha \geq 0$, 则 $\prod_{i=1}^{n} X_{i}$ 属于 $\mathcal{R}_{-\alpha}^{*}(\alpha \geq 0)$ 且

$$
\operatorname{Pr}\left(\prod_{i=1}^{n} X_{i}>x\right) \sim \sum_{i=1}^{n}\left(\prod_{j=1, j \neq i}^{n} E X_{j}^{\alpha}\right) \bar{F}_{i}(x)
$$

引理 2.2 与 Breiman's 定理形式上相似。当 $n=2$ 时, 引理 2.2 就得到最简单的形式:

$$
\operatorname{Pr}\left(X_{1} X_{2}>x\right) \sim E X_{1}^{\alpha} \bar{F}_{2}(x)+E X_{2}^{\alpha} \bar{F}_{1}(x) .
$$

\section{3. 主要结论及其证明}

为了得到我们的结果, 我们需要作如下假设:

假设 $\mathbf{a}$ : 由 $F, G$ 构成的凸组合是强正则变化族 $\left(\mathcal{R}_{-\alpha}^{*}\right)$, 即

$$
b F+(1-b) G \in \mathcal{R}_{-\alpha}^{*},
$$

其中 $b \in(0,1)$ 。事实上, 假设 a 包含以下 3 种情形, 具体如下 $[11]$ :

1) $F \in \mathcal{R}_{-\alpha}^{*}, \quad \bar{G}(x)=o(\bar{F}(x))$;

2) $F \in \mathcal{R}_{-\alpha}^{*}, \quad G \in \mathcal{R}_{-\alpha}$ 且 $\bar{G}(x)=O(\bar{F}(x))$; 
3) $F \in \mathcal{R}_{-\alpha}^{*}, G \in \mathcal{R}_{-\alpha}^{*}$ 且函数 $a(x)=\frac{\bar{F}\left(e^{x}\right)}{\bar{G}\left(e^{x}\right)}$, 对任意的 $y>0$, 有 $a(x y) \asymp a(x)$ 。

根据引言介绍, 我们知道研究带有保险风险和金融风险的破产概率问题, 实质上是研究随机变量乘 积的尾部渐近性。因此, 我们设两个随机变量 $X$ 与 $Y$, 这里 $X$ 表示保险风险, $Y$ 表示金融风险, 其对应 的边缘分布函数分别为 $F, G$ 且由随机变量 $X$ 与 $Y$ 构成的随机向量 $(X, Y)$ 服从广义 FGM 分布(2.1 式)。记 $H$ 表示随机向量 $(X, Y)$ 的乘积分布 ( $H$ 表示随机变量 $X Y$ 的分布), 我们的目标就是研究随机变量 $X$ 与 $Y$ 之 间的相依结构对其乘积尾分布 $\bar{H}$ 的影响。延续文献[7]等表示方法, 设随机向量 $\left(X^{*}, Y^{*}\right)$ 是随机向量 $(X, Y)$ 独立复制, 即随机向量 $\left(X^{*}, Y^{*}\right)$ 与随机向量 $(X, Y)$ 具有相同的分布但 $\left(X^{*}, Y^{*}\right)$ 具有独立分量, 于是, 记 $H^{*}$ 表示随机向量 $\left(X^{*}, Y^{*}\right)$ 的乘积分布 $\left(H^{*}\right.$ 表示随机变量 $X^{*} Y^{*}$ 的分布 $)$ 。最后, 在给出定理之前先引入两个 的新随机变量 $X_{A}$ 和 $Y_{B}$, 其对应分布函数分别为: $F_{A}, G_{B}$, 定义[10]:

$$
F_{A}(x) \triangleq F(x)[1-A(F(x))], G_{B}(y) \triangleq G(y)[1-B(G(y))], x, y \geq 0
$$

再设 $\eta_{A}, \eta_{B}$ 为函数 $A(u), B(v)$ 在 $u=1, v=1$ 时的左导数且 $\left|\eta_{A}\right| \vee\left|\eta_{B}\right| \leq 1$, 我们约定 $\left.\frac{A(u)}{1-u}\right|_{u=1}=-\eta_{A}$, $\left.\frac{B(v)}{1-v}\right|_{v=1}=-\eta_{B}$, 根据研究可推得如下结论。

定理 3.1 : 设两个随机变量 $X$ 与 $Y$, 其对应的边缘分布为 $F, G$, 由随机变量 $X$ 与 $Y$ 构成的随机向量 $(X, Y)$ 服从广义 FGM 分布(2.1 式), 若 $F, G$ 满足假设 a 且 $E Y^{\alpha}<\infty$, 则:

$$
\bar{H}(x) \sim\left[\left(1+\eta_{A} \theta\right) E Y^{\alpha}-\eta_{A} \theta E Y_{B}^{\alpha}\right] \bar{F}(x)+\left[\left(1+\eta_{B} \theta\right) E X^{\alpha}-\eta_{B} \theta E X_{A}^{\alpha}\right] \bar{G}(x) .
$$

证明：根据文献[10]的研究和广义 FGM 分布的定义式，给出的定义(3.1)式是合理的。现在设随机向 量 $\left(X_{A}^{*}, Y_{B}^{*}\right)$ 是 $\left(X_{A}, Y_{B}\right)$ 独立的情形, 即随机向量 $\left(X_{A}^{*}, Y_{B}^{*}\right)$ 与随机向量 $\left(X_{A}, Y_{B}\right)$ 具有相同分布且 $\left(X_{A}^{*}, Y_{B}^{*}\right)$ 具 有独立分量, 随机向量 $\left(X_{A}^{*}, Y_{B}^{*}\right)$ 与随机向量 $\left(X^{*}, Y^{*}\right)$ 也独立. 若 $E Y^{\alpha}$ 存在, 则 $E Y_{B}^{\alpha}$ 也存在, 证明见文献 [10]。根据(3.1)式可得:

$$
A(F(x))=1-\frac{F_{A}(x)}{F(x)}, B(G(y))=1-\frac{G_{B}(y)}{G(y)}, x, y \geq 0
$$

代入到(2.1)式可得：

$$
\Pi(\mathrm{d} s, \mathrm{~d} t)=(1+\theta) F(\mathrm{~d} s) G(\mathrm{~d} t)-\theta F_{A}(\mathrm{~d} s) G(\mathrm{~d} t)-\theta F(\mathrm{~d} s) G_{B}(\mathrm{~d} t)+\theta F_{A}(\mathrm{~d} s) F_{B}(\mathrm{~d} t)
$$

再对等式 3.3 两边积分可得：

$$
\begin{aligned}
\bar{H}(x) & =\operatorname{Pr}(X Y>x) \\
& =\iint_{s t>x, s>0, t>0} \prod(\mathrm{d} s, \mathrm{~d} t) \\
& =(1+\theta) \bar{H}^{*}(x)-\theta \operatorname{Pr}\left(X_{A}^{*} Y^{*}>x\right)-\theta \operatorname{Pr}\left(X^{*} Y_{B}^{*}>x\right)+\theta \operatorname{Pr}\left(X_{A}^{*} Y_{B}^{*}>x\right)
\end{aligned}
$$

对(3.4)式, 应用引理 2.2 得:

$$
\begin{aligned}
\bar{H}(x) \sim(1+\theta)\left[E Y^{\alpha} \bar{F}(x)+E X^{\alpha} \bar{G}(x)\right]-\theta\left[E Y^{\alpha} \operatorname{Pr}\left(X_{A}^{*}>x\right)+E X_{A}^{\alpha} \bar{G}(x)\right] \\
-\theta\left[E Y_{B}^{\alpha} \bar{F}(x)+E X^{\alpha} \operatorname{Pr}\left(Y_{B}^{*}>x\right)\right]+\theta\left[E Y_{B}^{\alpha} \operatorname{Pr}\left(X_{A}^{*}>x\right)+E X_{A}^{\alpha} \operatorname{Pr}\left(Y_{B}^{*}>x\right)\right]
\end{aligned}
$$

又根据 $F_{A}(x)$ 和 $G_{B}(x)$ 的定义可得:

$$
\bar{F}_{A}(x) \sim\left(1-\eta_{A}\right) \bar{F}(x), \quad \bar{G}_{B}(x) \sim\left(1-\eta_{B}\right) \bar{G}(x)
$$


把(3.6)式代入(3.5)式, 整理得:

$$
\bar{H}(x) \sim\left[\left(1+\eta_{A} \theta\right) E Y^{\alpha}-\eta_{A} \theta E Y_{B}^{\alpha}\right] \bar{F}(x)+\left[\left(1+\eta_{B} \theta\right) E X^{\alpha}-\eta_{B} \theta E X_{A}^{\alpha}\right] \bar{G}(x)
$$

即, 定理 3.1 成立。

定理 3.2: 设 $\left\{\left(X_{1}, Y_{1}\right),\left(X_{2}, Y_{2}\right), \cdots,\left(X_{n}, Y_{n}\right)\right\}$ 是独立同分布随机对序列并服从广义 FGM 分布(见(2.1) 式), $X_{i}, Y_{i}$ 的边缘分布分别为 $F, G$, 若 $F, G$ 满足假设 a 且 $E Y^{\alpha}<\infty$, 则:

$$
\psi(x, n)=\operatorname{Pr}\left(M_{n}>x\right) \sim A_{n} \bar{F}(x)+B_{n} \bar{G}(x)
$$

其中, $A_{n}=\left(\left(1+\eta_{A} \theta\right) E Y^{\alpha}-\eta_{A} \theta E Y_{B}^{\alpha}\right) \sum_{i=1}^{n}\left(E Y^{\alpha}\right)^{i-1}$,

$$
B_{n}=\sum_{i=1}^{n}\left(E Y^{\alpha}\right)^{i-1}\left[\left(1+\eta_{B} \theta\right) E\left(M_{n-i}+X_{n-i+1}\right)_{+}^{\alpha}-\eta_{B} \theta E\left(M_{n-i}+X_{A, n-i+1}\right)_{+}^{\alpha}\right] .
$$

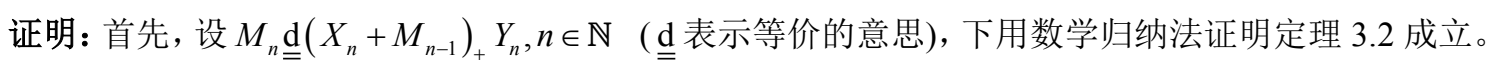

当 $n=1$ 时, 显然, $\psi(x, 1)=\operatorname{Pr}\left(M_{1}>x\right)=\operatorname{Pr}\left(X_{1,+} Y_{1}>x\right)=\bar{H}(x)$, 即 $n=1$ 成立;

现在假设 $n=n-1 \geq 1$ 时成立, 根据假设条件, 我们可知 $X_{n}, M_{n}, M_{n-1}, X_{n}+M_{n-1}, Y_{n}$ 都属于 $\mathcal{R}_{-\alpha}^{*} \subset \mathcal{S}(0)$ 。 应用引理 2.1(令 $\alpha=0)$, 可得:

$$
\operatorname{Pr}\left(X_{n}+M_{n-1}>x\right) \sim\left(1+A_{n-1}\right) \bar{F}(x)+B_{n-1} \bar{G}(x)
$$

于是, 结合 $M_{n}$ 的等价定义有:

$$
\begin{aligned}
\psi(x, n)= & \operatorname{Pr}\left(M_{n}>x\right)=\operatorname{Pr}\left(\left(X_{n}+M_{n-1}\right)_{+} Y_{n}>x\right) \\
= & (1+\theta) \operatorname{Pr}\left(\left(X_{n}^{*}+M_{n-1}\right)_{+} Y^{*}>x\right)-\theta \operatorname{Pr}\left(\left(X_{A, n}^{*}+M_{n-1}\right)_{+} Y^{*}>x\right) \\
& -\theta \operatorname{Pr}\left(\left(X_{n}^{*}+M_{n-1}\right)_{+} Y_{B}^{*}>x\right)+\theta \operatorname{Pr}\left(\left(X_{A, n}^{*}+M_{n-1}\right)_{+} Y_{B}^{*}>x\right)
\end{aligned}
$$

根据引理 2.1, 则(3.9)式等价于

$$
\begin{aligned}
\psi(x, n) \sim & (1+\theta)\left[E\left(X_{n}+M_{n-1}\right)_{+}^{\alpha} \bar{G}(x)+E Y^{\alpha} \operatorname{Pr}\left(\left(X_{n}^{*}+M_{n-1}\right)_{+}>x\right)\right] \\
& -\theta\left[E\left(X_{A, n}+M_{n-1}\right)_{+}^{\alpha} \bar{G}(x)+E Y^{\alpha} \operatorname{Pr}\left(\left(X_{A, n}^{*}+M_{n-1}\right)_{+}>x\right)\right] \\
& -\theta\left[E\left(X_{n}+M_{n-1}\right)_{+}^{\alpha} \operatorname{Pr}\left(Y_{B}^{*}>x\right)+E Y_{B}^{\alpha} \operatorname{Pr}\left(\left(X_{n}^{*}+M_{n-1}\right)_{+}>x\right)\right] \\
& +\theta\left[E\left(X_{A, n}+M_{n-1}\right)_{+}^{\alpha} \operatorname{Pr}\left(Y_{B}^{*}>x\right)+E Y_{B}^{\alpha} \operatorname{Pr}\left(\left(X_{A, n}^{*}+M_{n-1}\right)_{+}>x\right)\right]
\end{aligned}
$$

结合(3.6)式、(3.8)式, 计算得:

$$
\begin{aligned}
\psi(x, n) \sim[(1 & \left.\left.+\theta \eta_{A}\right) E Y^{\alpha}-\theta \eta_{A} E Y_{B}^{\alpha}+E Y^{\alpha} A_{n-1}\right] \bar{F}(x) \\
& +\left[\left(1+\theta \eta_{B}\right) E\left(X_{n}+M_{n-1}\right)_{+}^{\alpha}-\theta \eta_{B} E\left(X_{A, n}+M_{n-1}\right)_{+}^{\alpha}+E Y^{\alpha} B_{n-1}\right] \bar{G}(x)
\end{aligned}
$$

整理得:

$$
\psi(x, n)=\operatorname{Pr}\left(M_{n}>x\right) \sim A_{n} \bar{F}(x)+B_{n} \bar{G}(x) .
$$

即定理 3.2 成立。

定理 3.3: 设 $\left\{\left(X_{1}, Y_{1}\right),\left(X_{2}, Y_{2}\right), \cdots,\left(X_{n}, Y_{n}\right)\right\}$ 是独立同分布随机对序列并服从广义 FGM 分布(见(2.1) 式), $X_{i}, Y_{i}$ 的边缘分布分别为 $F, G$, 若 $F, G$ 满足假设 a 且 $E Y^{\alpha}<\infty$, 则 


$$
\psi(x, n)=\operatorname{Pr}\left(S_{n}>x\right) \sim A_{n} \bar{F}(x)+C_{n} \bar{G}(x)
$$

其中, $A_{n}=\left(\left(1+\eta_{A} \theta\right) E Y^{\alpha}-\eta_{A} \theta E Y_{B}^{\alpha}\right) \sum_{i=1}^{n}\left(E Y^{\alpha}\right)^{i-1}$,

$$
C_{n}=\sum_{i=1}^{n}\left(E Y^{\alpha}\right)^{i-1}\left[\left(1+\eta_{B} \theta\right) E\left(S_{n-i}+X_{n-i+1}\right)_{+}^{\alpha}-\eta_{B} \theta E\left(S_{n-i}+X_{A, n-i+1}\right)_{+}^{\alpha}\right] .
$$

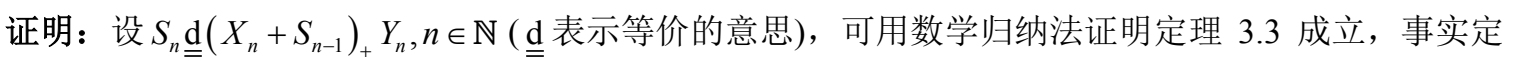
理 3.3 证明与定理 3.2 证明相类似, 这里都不再展开。

注记 3.1: 若我们取 $A(u)=1-u, B(v)=1-v$, 此时, $\eta_{A}=\eta_{B}=-1$, 则:

$$
\psi(x, n)=\operatorname{Pr}\left(M_{n}>x\right) \sim A_{n}^{\prime} \bar{F}(x)+B_{n}^{\prime} \bar{G}(x)
$$

和

$$
\psi(x, n)=\operatorname{Pr}\left(S_{n}>x\right) \sim A_{n}^{\prime} \bar{F}(x)+C_{n}^{\prime} \bar{G}(x)
$$

其中, $A_{n}^{\prime}=\left((1-\theta) E Y^{\alpha}+\theta E Y_{B}^{\alpha}\right) \sum_{i=1}^{n}\left(E Y^{\alpha}\right)^{i-1}$,

$$
\begin{gathered}
B_{n}^{\prime}=\sum_{i=1}^{n}\left(E Y^{\alpha}\right)^{i-1}\left[(1-\theta) E\left(M_{n-i}+X_{n-i+1}\right)_{+}^{\alpha}+\theta E\left(M_{n-i}+X_{A, n-i+1}\right)_{+}^{\alpha}\right], \\
C_{n}^{\prime}=\sum_{i=1}^{n}\left(E Y^{\alpha}\right)^{i-1}\left[(1-\theta) E\left(S_{n-i}+X_{n-i+1}\right)_{+}^{\alpha}+\theta E\left(S_{n-i}+X_{A, n-i+1}\right)_{+}^{\alpha}\right] .
\end{gathered}
$$

即 Li \& Tang [11]文中的推论 2.1(a)。

推论 3.1: 在定理 3.2 的前提下, 如果 $E Y^{\alpha}<1$, 则 $n \rightarrow \infty$ 有:

$$
\psi(x)=\operatorname{Pr}\left(M_{\infty}>x\right) \sim A_{\infty} \bar{F}(x)+B_{\infty} \bar{G}(x)
$$

其中, $A_{\infty}=\frac{1}{1-E Y^{\alpha}}\left(\left(1+\eta_{A} \theta\right) E Y^{\alpha}-\eta_{A} \theta E Y_{B}^{\alpha}\right)$,

$$
B_{\infty}=\frac{1}{1-E Y^{\alpha}}\left[\left(1+\eta_{B} \theta\right) E\left(M_{\infty}+X\right)_{+}^{\alpha}-\eta_{B} \theta E\left(M_{\infty}+X_{A}\right)_{+}^{\alpha}\right] .
$$

推论 3.2: 在定理 3.3 的前提下, 如果 $E Y^{\alpha}<1$ 且 $E \ln \left(X_{-} \vee 1\right)<\infty$, 则 $n \rightarrow \infty$ 有:

$$
\psi(x)=\operatorname{Pr}\left(S_{n}>x\right) \sim A_{\infty} \bar{F}(x)+C_{\infty} \bar{G}(x)
$$

其中, $A_{\infty}=\frac{1}{1-E Y^{\alpha}}\left(\left(1+\eta_{A} \theta\right) E Y^{\alpha}-\eta_{A} \theta E Y_{B}^{\alpha}\right)$,

$$
C_{\infty}=\frac{1}{1-E Y^{\alpha}}\left[\left(1+\eta_{B} \theta\right) E\left(S_{\infty}+X\right)_{+}^{\alpha}-\eta_{B} \theta E\left(S_{\infty}+X_{A}\right)_{+}^{\alpha}\right] .
$$

注记 3.2: 在推论 3.1, 3.2 中, 若 $A(u)=1-u, B(v)=1-v$, 则推论 3.1, 3.2 即 Li\&Tang [11]文中对 应的推论 2.1(b)(c)。

\section{参考文献 (References)}

[1] Tang, Q. and Tsitsiashvili, G. (2003) Precise Estimates for the Ruin Probability in Finite Horizon in a Discrete-Time Model with Heavy-Tailed Insurance and Financial Risks. Stochastic Processes and Their Applications, 108, 299-325. http://dx.doi.org/10.1016/j.spa.2003.07.001

[2] Liu, F. (2013) Heavy-Tailed Distribution in the Presence of Dependence in Insurance and Finance. Doctoral Disserta- 
tion, University of Liverpool, Liverpool. http://repository.liv.ac.uk/14419/

[3] Vervaat, W. (1979) On a Stochastic Difference Equation and a Representation of Non-Negative Infinitely Divisible Random Variables. Advances in Applied Probability, 11, 750-783. http://www.jstor.org/stable/1426858

[4] Tang, Q. and Tsitsiashvili, G. (2004) Finite- and Infinite-Time Ruin Probabilities in the Presence of Stochastic Returns on Investments. Advances in Applied Probability, 36, 1278-1299. http://dx.doi.org/10.1239/aap/1103662967

[5] Chen, Y.Q. and Xie, X.S. (2005) The Finite Time Ruin Probability with the Same Heavy-Tailed Insurance and Financial Risks. Acta Mathematicae Applicatae Sinica, 21, 153-156. http://link.springer.com/article/10.1007\%2Fs10255-005-0226-y

[6] Zhang, Y., Shen, X. and Weng, C. (2009) Approximation of the Tail Probability of Randomly Weighted Sums and Applications. Stochastic Processes and Their Applications, 119, 655-675. http://dx.doi.org/10.1016/j.spa.2008.03.004

[7] Chen, Y. (2011) The Finite-Time Ruin Probability with Dependent Insurance and Financial Risks. Journal of Applied Probability, 48, 1035-1048. http://dx.doi.org/10.1239/jap/1324046017

[8] Chen, Y., Liu, J. and Liu, F. (2015) Ruin with Insurance and Financial Risks Following the Least Risky FGM Dependence Structure. Insurance: Mathematics and Economics, 62, 98-106. http://dx.doi.org/10.1016/j.insmatheco.2015.03.007

[9] Yi, L., Chen, Y. and Su, C. (2011) Approximation of the Tail Probability of Randomly Weighted Sums of Dependent Random Variables with Dominated Variation. Journal of Mathematical Analysis and Applications, 376, $365-372$. http://dx.doi.org/10.1016/j.jmaa.2010.10.020

[10] Jiang, J. and Tang, Q. (2011) The Product of Two Dependent Random Variables with Regularly Varying or Rapidly Varying Tails. Statistics \& Probability Letters, 81, 957-961. http://dx.doi.org/10.1016/j.spl.2011.01.015

[11] Li, J. and Tang, Q. (2015) Interplay of Insurance and Financial Risks in a Discrete-Time Model with Strongly Regular Variation. Bernoulli, 21, 1800-1823. http://arxiv.org/pdf/1507.07673.pdf

[12] 杨洋, 王开永. 保险风险管理中的破产渐近分析[M]. 北京: 科学出版社, 2013.

[13] Embrechts, P., Klüppelberg, C. and Mikosch, T. (2013) Modelling Extremal Events: For Insurance and Finance. Vol. 33, Springer Science \& Business Media, Berlin.

[14] Nelsen, R.B. (2007) An Introduction to Copulas. Springer Science \& Business Media, Berlin. 